\title{
The HDAC inhibitor phenylbutyrate reverses effects of neonatal ventral hippocampal lesion in rats
}

\author{
Guy Sandner ${ }^{1}$, Lionel Host ${ }^{2}$, Marie-Josée Angst ${ }^{1}$, Thierry Guiberteau ${ }^{3}$, Blandine Guignard ${ }^{3}$ and Jean Zwiller ${ }^{2}$ \\ 1 U666 INSERM, Faculté de Médecine, Université de Strasbourg, Strasbourg, France \\ 2 UMR 7237, Centre National de la Recherche Scientifique, Faculté de Psychologie, Université de Strasbourg, Strasbourg, France \\ ${ }_{3}$ UMR 7191, Centre National de la Recherche Scientifique/Université Louis Pasteur, Faculté de Médecine, Université de Strasbourg, Strasbourg, France
}

\section{Edited by:}

Bernat Kocsis, Harvard Medical School,

USA

\section{Reviewed by:}

Kuei-Yuan Tseng, Rosalind Franklin University of Medicine and Science, USA

Robert Chambers, Indiana University

School of Medicine, USA

Dennis Grayson, University of Illinois at

Chicago, USA

${ }^{*}$ Correspondence:

Guy Sandner, U666 INSERM, Faculté de médecine, Université de

Strasbourg, 11 rue Humann, 67085

Strasbourg Cedex, France.

e-mail: sandner@neurochem.u-strasbg.fr
Recent evidence suggests that epigenetic mechanisms play a role in psychiatric diseases. In this study, we considered rats with neonatal ventral hippocampal lesions (NVHL) that are currently used for modeling neurodevelopmental aspects of schizophrenia. Contribution of epigenetic regulation to the effects of the lesion was investigated, using a histone deacetylase (HDAC) inhibitor. Lesioned or sham-operated rats were treated with the general HDAC inhibitor phenylbutyrate, which was injected daily from the day after surgery until adulthood. Changes in the volume of the lesion were monitored by magnetic resonance imaging (MRI). Anxiety was analyzed in the Plus Maze Test. Hypersensitivity of the dopaminergic system was evaluated by measuring the locomotor response to apomorphine. An associative conditioning test rewarded with food was used to evaluate learning abilities. The volume of the lesions expanded long after surgery, independently of the treatment, as assessed by MRI. Removal of the ventral hippocampus reduced anxiety, and this remained unchanged when animals were treated with phenylbutyrate. In contrast, NVHL rats' hypersensitivity to apomorphine and deterioration of the associative learning were reduced by the treatment. Global HDAC activity, which was increased in the prefrontal cortex of lesioned non-treated rats, was found to be reversed by HDAC inhibition. The study provides evidence that chromatin remodeling may be useful for limiting behavioral consequences due to lesioning of the ventral hippocampus at an early age. This represents a novel approach for treating disorders resulting from insults occurring during brain development.

Keywords: animal model of schizophrenia, ventral hippocampal lesion, histone deacetylase inhibitor, brain ontogenesis, epigenetic regulation

\section{INTRODUCTION}

A number of studies have highlighted the importance of the epigenetic control of gene transcription in psychiatric disorders, including schizophrenia (Gavin and Sharma, 2010). Epigenetic control involves mechanisms that regulate gene transcription by modifying the accessibility of genes to the transcriptional machinery (Szyf et al., 2008), which is achieved via complex mechanisms, in which DNA methylation and post-translational modifications of histones play a major role (Lopez-Rodas et al., 1993). Increased DNA methylation is associated with reduced transcriptional activity related to tighter DNA coiling. Reduced transcriptional activity may also result from histone deacetylation brought about by histone deacetylases (HDACs). HDAC activity was found to be enhanced in patients with schizophrenia, especially in the prefrontal cortex (Roth et al., 2009). The development of a series of HDAC inhibitors as tools for modifying chromatin remodeling has provided a unique opportunity for the pharmacological handling of epigenetic regulation. The compounds have been shown to modify brain functioning, and to subsequently alter behavior. For instance, we have demonstrated that repeated treatment with several HDAC inhibitors reduced the motivation of rats to self-administer cocaine (Romieu et al., 2008). Others demonstrated that it reversed learning deficits including those related to aging (Fischer et al., 2007; Dash et al., 2009; Ricobaraza et al., 2009; Kilgore et al., 2010; Peleg et al., 2010).

Neonatal lesioning of the ventral hippocampus (NVHL) in rats produces an animal model currently used for documenting the neurodevelopmental hypothesis of schizophrenia (reviews in: Lipska, 2004; Tseng et al., 2009). The lesion performed at the end of the first week of life can be checked and its progression monitored using magnetic resonance imaging (MRI; Angst et al., 2007; Macedo et al., 2008, 2010; Bertrand et al., 2010; Sandner et al., 2010). Even if no major damage to the ventral hippocampus was found in patients, the approach nevertheless remains relevant since it triggers neurodevelopmental changes in the dopaminergic mesocorticolimbic system and in the prefrontal cortex, which are now considered central to the model (Halim and Swerdlow, 2000). The sensitivity of lesioned animals to dopaminergic agents was enhanced and a number of basic emotional reactions and cognitive abilities were modified (Tseng et al., 2009).

The hippocampus and prefrontal cortex contribute to several cognitive processes including awareness, attention, sensory information processing, hedonic evaluation, and memory (Nauta, 1971; Broersen, 2000; Rolls, 2000). These brain areas are also involved in the management of fear and anxiety (Shah and Treit, 2003; Degroot 
and Treit, 2004). All these aspects are relevant for schizophrenia. In the present study, a fearless associative learning task was conducted for testing awareness and memory (Holland and Petrovich, 2005; Macedo et al., 2008). Fear or anxiety was evaluated using the classical plus maze test (Pellow and File, 1986). A D2 agonist, apomorphine, was administered to challenge the dopaminergic system which results in hyperlocomotion. Apomorphine was used rather than amphetamine for the following reasons: (1) it has been used in our previous studies (Macedo et al., 2008); (2) classical neuroleptic drugs show D2 antagonistic properties (Ellenbroek and Cools, 2002); (3) amphetamine modifies less specifically the activity of dopaminergic neuronal pathways (Schmitt and Reith, 2010); (4) observations based on amphetamine-elicited hyperlocomotion may be beset with sensitization (Featherstone et al., 2007); and (5) addiction to amphetamine, being itself sensitive to HDAC inhibitors, may interfere with the observations (Romieu et al., 2008).

The present study aims at ascertaining whether repeated treatment with the HDAC inhibitor phenylbutyrate, administered from the day after the lesion until adulthood, would be able to counteract some of the characteristic anatomical and functional brain impairments found in NVHL rats.

\section{MATERIALS AND METHODS}

The methodology and protocols were approved by the French regional committee of ethics (CREMEAS) under the reference AL/01/19/10/08. All the procedures complied with the 1986 European Council Directive (86/609/EEC), itself being in agreement with the "Declaration of Helsinki."

\section{ANIMALS \\ Sprague Dawley dam rats were purchased with their pups from Charles River, France, and housed on a 14/10-h light/dark cycle (lights on at $7 \mathrm{AM}$ ) with food and water available ad libitum, except during the conditioning experiment. The pups were submitted to a lesion or sham operation at the age of 7 days. MRI picture acqui- sitions were performed at 3, 8, and 20 weeks, i.e., respectively in juvenile, young, and senior adults. Behavioral tests were conducted at the age of 10-20 weeks. The rats were killed in the week after the final MRI session. Brain sections were prepared and submitted to histology so that lesion size could be assessed, and the prefrontal cortex was dissected for biochemical investigation.}

\section{SURGERY}

Surgical procedures were performed at PN7 under isoflurane anesthesia as detailed previously (Angst et al., 2007) and were similar to those used by others for the model (Tseng et al., 2009). Either $0.3 \mu \mathrm{L}$ of ibotenic acid (Sigma, France, $10 \mu \mathrm{g} / \mu \mathrm{L}, \mathrm{pH} 7.4$ ) in the case of "NVHL rats," or artificial cerebrospinal fluid in the case of "sham-operated rats" was infused bilaterally into the ventral hippocampus. Three weeks after surgery, pups were weaned and housed two per cage.

\section{TREATMENT}

Sodium 4-phenylbutyrate $(20 \mathrm{mg} / \mathrm{kg}$ ) prepared in phosphate buffered saline (PBS, pH 7.4) was injected subcutaneously on a daily basis, starting with the day after surgery for the lesion until the end of week 9 (adulthood), to 17 lesioned and 9 sham-operated rats.
Vehicle was likewise injected in 17 lesioned and 9 sham-operated animals. Rats were weighed twice a week so that the amounts to be injected could be adjusted. The rats were weighed again prior to euthanasia, at 21 weeks of age.

\section{IMAGING TECHNIOUES, MRI}

Lesioned rats were subjected to three MRI sessions, at 3, 8, and 20 weeks of age under isoflurane anesthesia. MRI was performed on a scanner dedicated to small animals operating at $4.7 \mathrm{~T}$ (TR/TE/ TEeff: $3000 / 30 \mathrm{~ms} / 60 \mathrm{~ms})$. A series of 10 slices $(512 \times 512$ pixels $)$ was generated over a $1-\mathrm{cm}$-long section of the brain rostral to the cerebellum-cerebrum gap. The first MRI session served to select rats for the subsequent experiments. Rats presenting a moderate bilateral lesion were included in the study. We used a full $2 \times 2$ experimental design comprising nine rats per group. More specifically, we included nine lesioned rats treated with phenylbutyrate and nine lesioned rats that received vehicle. Two groups of nine sham lesioned groups, which received either phenylbutyrate or vehicle, were considered as controls. The other MRI sessions served to evaluate the broadening of the lesions with age. Lesioned areas were drawn on brain MRI coronal slices size-adjusted to compensate for brain growth. The numbers of pixels of left and right lesion drawings were added together. They represent the volume estimates on which ANOVA was computed, with age as a within-group factor, and lesion and treatment as two between-group factors. For graphical representation purposes, lesion volume was divided by estimated brain volume for the sake of homogeneity with a previous study (Macedo et al., 2010).

\section{LEARNING TEST}

Rats were subjected to a conditioning procedure (Holland and Petrovich, 2005) which had been adapted to characterize the cognitive effect of neonatal ventral hippocampal lesions (Macedo et al., 2008).Three days prior to the beginning of the procedure, rats were deprived of food during the dark period. A recessed food magazine was placed in a wall of an experimental chamber $(25 \mathrm{~cm} \times 35 \mathrm{~cm})$. It was crossed by an infrared photo-beam to detect nose pokes. A light visual cue and a loud speaker were placed above it. A microcomputer delivered cues or a food reward (two $45 \mathrm{mg}$ food pellets, Noyes rodent food pellets, Formula P, New Brunswick, NJ, USA) and recorded the periods of photo-beam interruption. Rats were first trained to feed from the food magazine. During this preliminary session, no cue was used and the reward was given randomly five times during a 30-min-period. Rats were then subjected to 8 days of two $30 \mathrm{~min}$-conditioning sessions, one in the morning and the other one in the afternoon. In each session, rats received either five presentations of a cue, light, or tone, lasting $10 \mathrm{~s}$, immediately followed by delivery of the reward (CS+ condition). They were also submitted to a control condition that consisted in five presentations of the other stimulus, with the food delivery system being switched off (CS- condition). All conditions were balanced: (1) the computer controlled four cages simultaneously, each one containing a rat belonging to each of the four groups; (2) for half the rats, the CS+ was the tone, and for the other half it was the light; (3) the morning/afternoon CS+/CS- sessions were spread randomly across the groups. The average nose-poke duration during the five last seconds of cue presentation was recorded and was considered 
an index of anticipation of the reward (Holland and Petrovich, 2005). It constituted the dependent variable of an ANOVA, where the associative feature (CS+ vs. CS-) and series of daily sessions were two within-group factors, and the lesion and treatment being two between-group factors.

\section{LOCOMOTION UNDER APOMORPHINE}

Locomotion was evaluated in eight identical test cages $(30 \mathrm{~cm} \times 40 \mathrm{~cm})$. They were different from the home cages in many respects (different shape, dimmer light, silent environment, walls with black stripes). A passive infrared detector (IRP 124, Talco, France) was used to monitor activity during consecutive 5 minperiods over $1 \mathrm{~h}$. The detector comprised a gallium-arsenide infrared sensitive surface placed behind a Fresnel lens and reacted to the heat emitted by the rat. The lens determined $8 \times 3$ sectors. Each transition from one sector to another provided an electric pulse to the computer interface. Measurements started immediately after the rats had been subcutaneously injected with an apomorphine solution prepared in saline $(0.35,0.70$, or $1.40 \mathrm{mg} /$ $\mathrm{kg}$ ). In order to minimize sensitization, rats were tested at increasing dosage, and the successive tests were separated by at least a 2-week-period. Results were submitted to an ANOVA, with the successive series of 5 min measurements and the dose of apomorphine as two within-group factors, and the lesion and treatment as two between-group factors. Noteworthy, all rats were subjected to the same treatment schedule so that any differential result in the following test cannot be attributed to the effect of apomorphine per se.

\section{ANXIETY, ELEVATED PLUS MAZE TEST}

As a last behavioral experiment, the rats were subjected to the standard Elevated Plus Maze Test for anxiety (Pellow and File, 1986). It is made of two closed arms (i.e., surrounded by walls), orthogonal to two open arms. The rats were placed on a central platform facing an open arm. Their position was recorded for $30 \mathrm{~min}$. The time spent in the open arms and the number of entries were submitted to ANOVAs, with the arms (open vs. closed) as a within-group factor, and the lesion and treatment as two between-group factors.

\section{POST-MORTEM HISTOLOGY AND HDAC TITRATION}

The rats were given an overdose of pentobarbital ( $1 \mathrm{~mL}$ Doléthal, Vétoquinol, France). Their brain was removed and a 3-mm-thick slice cut away, the upper surface being $1 \mathrm{~mm}$ rostral to the corpus callosum (coronal slice extending from Bregma -0.3 to $2.7 \mathrm{~mm}$ ). This slice was then placed on a stainless steel plate at $4^{\circ} \mathrm{C}$, and the left medial prefrontal cortical area containing the pre- and infra-limbic cortex, the cingulate cortex, and part of the secondary motor cortex was cut out. It was immediately immersed into liquid nitrogen and processed for HDAC activity measurement the next day. The remaining caudal brain was fixed by immersion in $4 \%$ formalin and used to verify the lesion on 25 - $\mu$ m-thick sections stained with Cresyl violet. Total nuclear HDAC activity was measured with the Fluor de Lys assay system (Biomol, Plymouth, PA, USA), as described previously (Romieu et al., 2008). Deacetylase activity was expressed as arbitrary fluorescence units per minute per milligram of protein and subjected to an ANOVA, with the lesion and treatment as two between-group factors.

\section{RESULTS}

\section{BODY WEIGHT}

Neonatal ventral hippocampal lesions rats weighed less than shamoperated rats: The effect of lesion and of the interaction between lesion and age were significant [respectively $F(1,32)=7.00, p<0.05$ and $F(6,192)=3.09, p<0.01]$, which indicates reduced growth of NVHL rats. Differences in body weight could still be detected in 21-week-old rats, with NVHL rats about 10\% lighter than their control littermates [lesioned rats, 501 g; sham operated, 549 g; $F(1,32)=5.73, p<0.05]$. The treatment had no statistically significant effect on body weight.

\section{EVOLUTION OF THE LESIONED BRAIN AREAS}

Photomicrographs representing MRI images of a typical lesioned area at 3, 8, and 20 weeks are shown at the bottom of Figure 1. Figure 1 also shows the relative volumes of the lesions at these ages. The relative volume of the lesion expanded significantly as the rats aged $[F(2,32)=31.0, p<0.0001]$. No statistically significant effect of the treatment was found $[F(1,16)=1.57]$, indicating that there was no selection bias. Interaction with age was close to the significance threshold $[F(2,32)=3.17, p=0.056]$.

\section{ASSOCIATIVE LEARNING}

Results of the associative learning experiments, expressed as nosepoke durations at the end of the rewarded stimulus for the anticipation of the reward (CS+), are shown in Figure 2. Values for the CS- control group remained close to baseline and are therefore not illustrated. Mean nose-poke durations increased between sessions in all groups. It took more sessions for the untreated NVHL group to learn that the cue predicted food. Administering phenylbutyrate had no effect on the learning process in the sham-operated groups of rats. NVHL rats treated with phenylbutyrate attained a steady level that was higher than that attained by vehicle-injected NVHL rats, and in fewer sessions. The two criteria for adequate learning were statistically met: there was a difference according to the whether or not food was announced by the stimulus [CS+ vs.

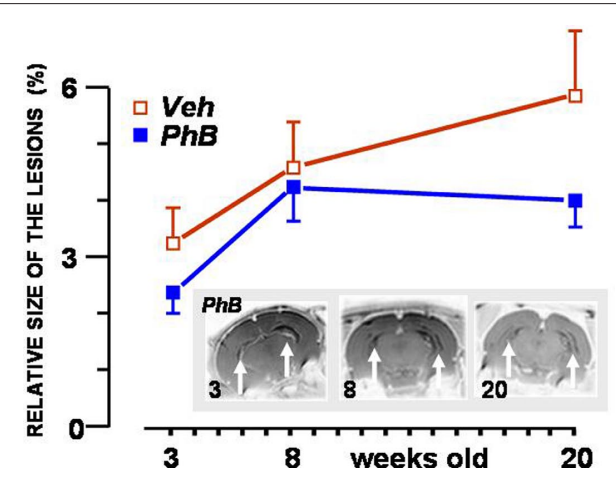

FIGURE 1 | Volume of the neonatal ventral hippocampal lesion expressed relative to brain size at the age of $\mathbf{3 , 8}$, and $\mathbf{2 0}$ weeks. Values represent means $( \pm$ SEM) of the relative volume of the lesion in rats that were treated with $20 \mathrm{mg} / \mathrm{kg}$ phenylbutyrate (PhB: blue squares) or the same volume of the vehicle PBS (Veh: open red squares). Photomicrographs at the bottom show three RMI pictures of the lesion at the three ages considered, taken from a rat belonging to the phenylbutyrate-treated group of rats. Lesioned areas are marked with arrows. 


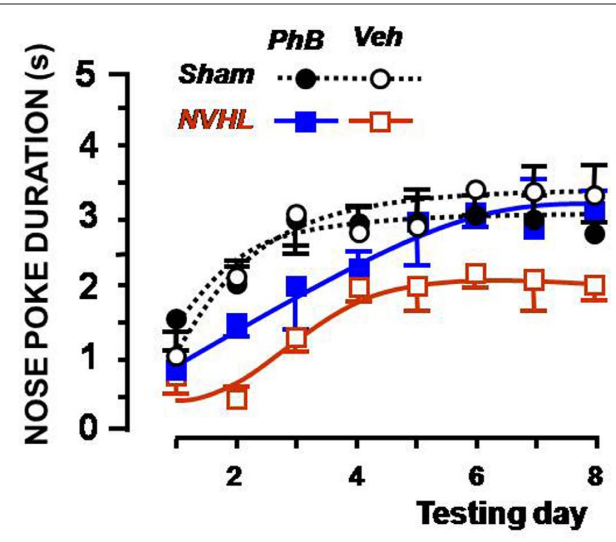

FIGURE 2 | Effect of the phenylbutyrate treatment on lesioned rats in the associative learning test. The graph shows the duration of nose poking in the food magazine during the 5 -s before food was delivered (ceiling $=5 \mathrm{~s}$ ). The $X$-axis depicts the sequence of daily training sessions in which $10 \mathrm{~s}$ of the cue preceded food delivery. The $Y$-axis presents the mean (and SEMs) nose-poke duration for each daily session. Sham-operated rats (dotted lines) were treated with $20 \mathrm{mg} / \mathrm{kg}$ phenylbutyrate (dark circles) or vehicle (open circles). Lesioned rats (full lines) were treated with phenylbutyrate (blue squares) or PBS (open red squares).

CS-: $F(1,32)=63.8, p<0.0001]$, and a progression was observed from session to session $[F(7,224)=21.0, p<0.0001]$. The global effect of the lesion was significant $[F(1,32)=7.06, p<0.05)$ and interacted with the effect of treatment $[F(1,32)=5.03, p<0.05)$. This is evidence that the treatment was more or only efficient in NVHL rats, as seen in Figure 2. Interaction between the effect of the lesion and the changes between the sessions was also significant $[F(7,224)=2.81, p<0.01)$, which indicates that the learning process did not follow the same timeline in lesioned rats as in their shamoperated controls. It is noteworthy that the mean final performance of non-treated NVHL rats was not as good as the other groups. This had been already observed previously (Macedo et al., 2008).

\section{APOMORPHINE INDUCED LOCOMOTION}

No difference was noticed for the lowest apomorphine dose $(0.35 \mathrm{mg} / \mathrm{kg})$. Figure 3 shows the locomotion observed during $1 \mathrm{~h}$ after subcutaneous injection of the highest dose of apomorphine $(1.4 \mathrm{mg} / \mathrm{kg})$. For this apomorphine dose, the non-treated NVHL group displayed high activity, whereas the NVHL group of rats treated with phenylbutyrate showed substantially the same level of activity as the control groups. The test's timeline was significant $[F(11,352)=25.7, p<0.0001]$, and lesion interacted significantly with it $[F(11,352)=2.36, p<0.01)$, as well as with the apomorphine dose $[F(22,704)=6.96, p<0.0001]$. Statistical analysis also revealed a significant global effect of lesion $[F(1,32)=4.38, p<0.05]$, and of the apomorphine dose $[F(2,64)=14.7, p<0.0001]$ and a significant interaction between the two factors $[F(2,64)=3.58, p<0.05]$. These data denote a dose-dependent differential reactivity to apomorphine between lesioned and control rats, typical of this animal model. They also reveal a significant triple interaction between lesion, dose of apomorphine, and treatment $[F(2,64)=4.99$, $p<0.01$ ], demonstrating a differential reactivity to apomorphine when NVHL rats were administered phenylbutyrate.

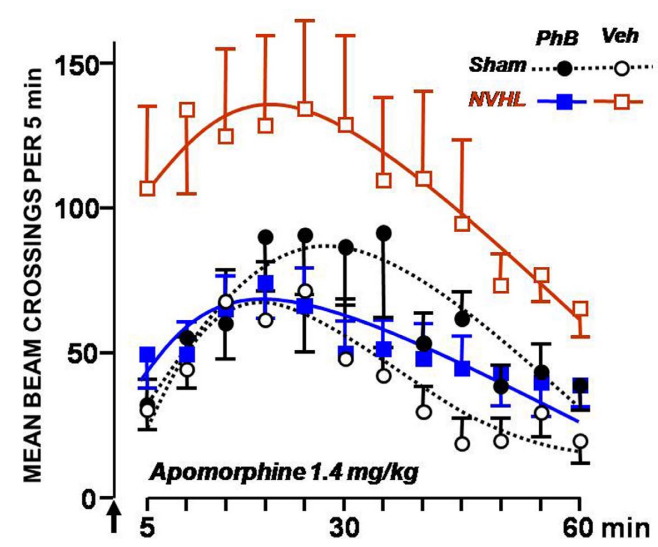

FIGURE 3 | Effect of the phenylbutyrate treatment on the locomotor activity elicited by apomorphine. Locomotor activity corresponding to beam crossings was plotted every 5 min following a subcutaneous injection of $1.4 \mathrm{mg} / \mathrm{kg}$ apomorphine with SEMs. The symbols used are those described in the legend to Figure 2. The arrow marks the time of injection.

\section{ELEVATED PLUS MAZE}

Results from the Elevated Plus Maze test are shown in Figure 4. Compared with the control groups, rats from the NVHL group spent more time in the open arms and went into them more frequently. As for the time spent in the open arms, the significant factors were the lesion $[F(1,32)=10.6, p<0.01]$, the arms $[F(1,32)=9.43, p<0.01]$, and the interaction between the two factors $[F(1,32)=6.22, p<0.05]$. Treatment with the HDAC inhibitor did not influence the test results $[F(1,32)=0.75]$. Concerning the number of open arm entries, the significant factors were the lesion $[F(1,32)=7.02, p<0.05]$, the arms $[F(1,32)=4.99, p<0.05]$, and the interaction between the two factors $[F(1,32)=9.55, p<0.01]$ The treatment had no influence on the results $[F(1,32)=0.01]$.

\section{HDAC ASSAY}

Figure 5 illustrates the global HDAC activity in nuclear extracts derived from the prefrontal cortex of the same four groups of rats. HDAC activity was found to be increased in lesioned vehicle-treated rats. Repeated treatment with phenylbutyrate restored activity to the level found in sham-operated animals. A significant interaction was found between the lesion and the treatment $[F(1,30)=5.10$, $p<0.05)$. It is worth noting that both the lesion and the treatment factors revealed a significant effect per se $[F(1,30)=9.86, p<0.01$ and $F(1,30)=13.4, p<0.001$, respectively].

\section{HISTOLOGY}

Figure 6 shows that there was no selection bias between phenylbutyrate and vehicle treated lesioned rats. The ventral hippocampus appeared to be severely damaged, except for dorsal and caudal ventral endings. Neither the dorsal hippocampus, nor the entorhinal cortex was damaged.

\section{DISCUSSION}

Histone deacetylase inhibition in rats reduced several long-term consequences of a neonatal ventral hippocampal lesion, such as provoked locomotion and alteration of a rewarded learning, but not 

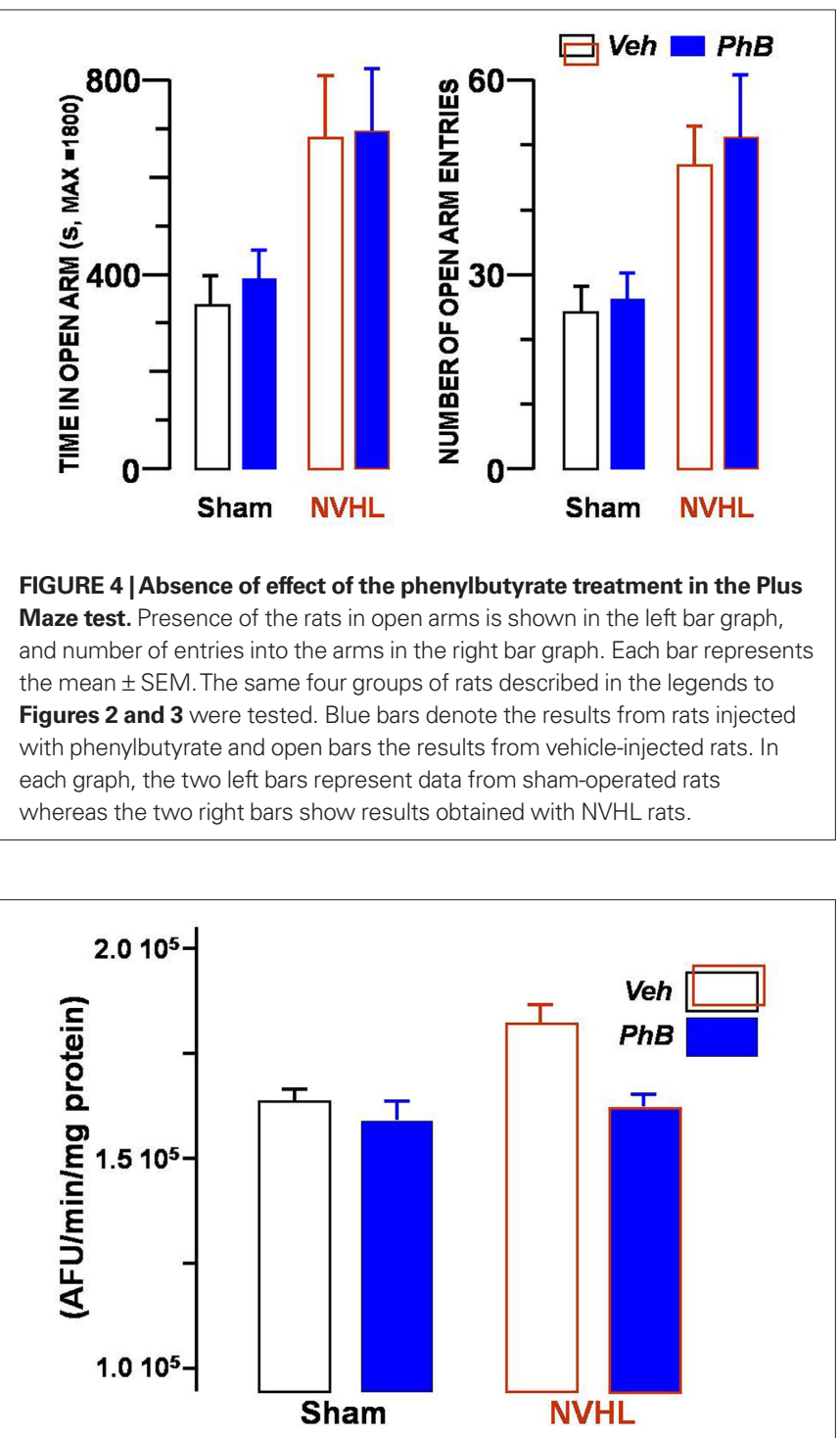

FIGURE 5 | Effect of phenylbutyrate on nuclear HDAC activity in the prefrontal cortex. Total HDAC activity was measured in the nuclear fraction prepared from samples of prefrontal cortex, using a fluorescent activity assay. Each bar represents the mean \pm SEM of arbitrary fluorescence units per minute and per milligram of protein. Blue bars show the results obtained from rats injected with phenylbutyrate, and open bars from vehicle-injected rats.

other effects of the lesion, such as reduced anxiety or anatomical changes to the brain. These are well documented outcomes of the lesion. Confirming such earlier observations ensures that there was no bias in our experimental strategy. We also replicated some of the less documented effects, confirming and expanding them. For instance, we confirmed that the size of the lesion increased until puberty (Bertrand et al., 2010; Sandner et al., 2010). The lesions continued extending after puberty, albeit at a lower rate.

\section{EVOLUTION OF THE LESIONS}

The broadening of the lesioned area has been attributed to the degeneration of other brain areas as an indirect consequence of the lesion (Halim and Swerdlow, 2000; Sandner et al., 2010), which can

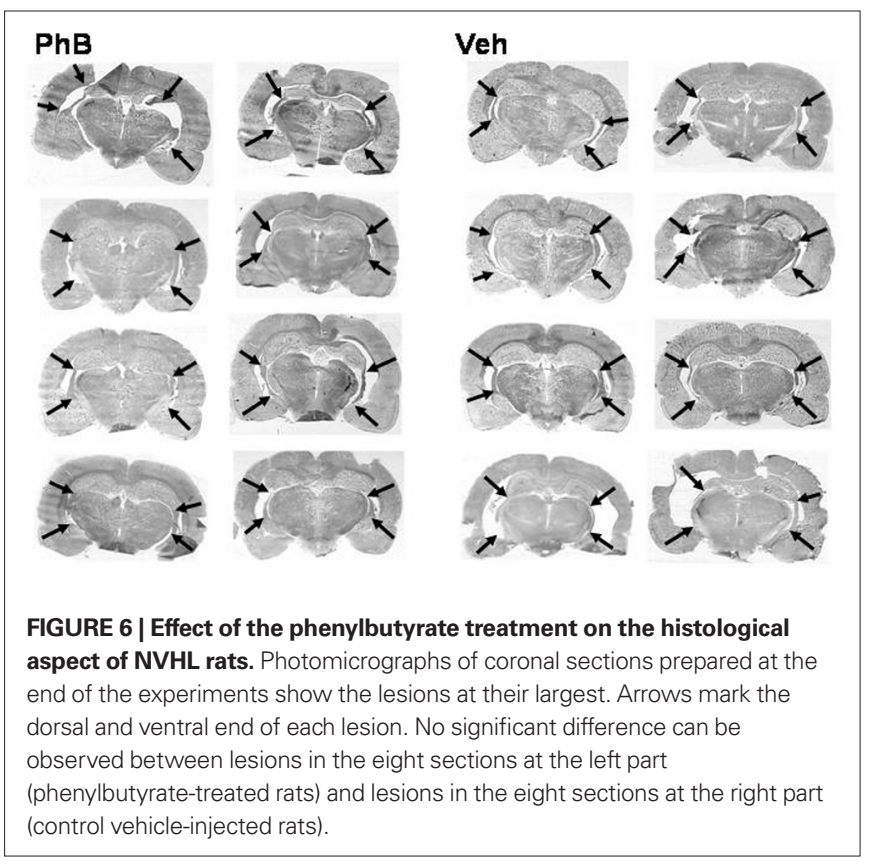

be compared to the gradual cortical shrinkage observed in some elderly schizophrenic patients (Cahn et al., 2009). From a theoretical point of view, it indicates that the NVHL model comprises a gradual degenerative aspect. It is worth noting that the effect of the treatment with phenylbutyrate nearly reached statistical significance in slowing down this evolution. The possibility that the treatment affected this macroscopic criterion of brain atrophy can therefore not be ruled out and deserves further investigation.

\section{EMOTIONAL REACTIVITY}

In the Elevated Plus Maze Test, rats spent more time in the open arms and went more often into them than their controls, which is usually interpreted as denoting reduced anxiety (Pellow and File, 1986). Reduced anxiety appears to be a general issue following damage to the neuronal network to which the ventral hippocampus belongs (Shah and Treit, 2003; Wood et al., 2003; Beninger et al., 2009). Even when the network was temporally switched off, for instance by means of tetrodotoxin injection into the ventral hippocampus, rats were less sensitive to fear (Degroot and Treit, 2004). Since similar anxiolytic effects were also reported when the medial prefrontal cortex was lesioned (Shah and Treit, 2003), one can assume that optimal management of emotions requires the integrity of the hippocampus-prefrontal cortex interplay that had been damaged and was neither spontaneously repaired nor with the treatment.

\section{HYPERLOCOMOTION UNDER APOMORPHINE}

It has been well documented that apomorphine elicits hyperlocomotion in NVHL rats (Schroeder et al., 1999). At the doses used here, some repetitive behavioral activities were observed in addition to mere locomotion. They consisted of repetitive oro-facial activity (sniffing around the edges of the cage, licking its bottom, carrying, and gnawing droppings), i.e., activity that was adapted to targets present in the cage, which is not observed in the case 
of amphetamine-induced stereotypy. It would probably be worth studying the ontogenesis of such behaviors in NVHL rats, as they were considered to represent developmental landmarks (Lepekhina and Tsitsurina, 2007) and the hypersensitive locomotor activity in response to dopaminergic agents appears only when rats become adult (Flores et al., 1996; Wood et al., 1997). At the dose of $0.7 \mathrm{mg} / \mathrm{kg}$ apomorphine, hyperlocomotion was only partially reversed by the phenylbutyrate treatment, whereas it was totally reversed for the higher dose of $1.4 \mathrm{mg} / \mathrm{kg}$. This somewhat paradoxical observation might tentatively be explained by two factors that could have affected the results obtained for the highest dose: hyperlocomotion was increased in all the groups boosting the baselines and excessive stereotypy may have reduced locomotion. Hypersensitivity to dopaminergic agents occurs when the prefrontal cortex is deprived of its hippocampal afferent neurons (Lipska et al., 1998). Regulation of neuronal activity in the nucleus accumbens by the prefrontal cortex is modified, resulting in the alteration of the sensitivity to dopaminergic drugs (Goto and O'Donnell, 2004). This behavioral aspect and the previously discussed associative conditioning were overcome by administering phenylbutyrate most probably because of the plasticity occurring in the still immature frontal cortex. This plasticity has already been successfully reversed, but with different means, namely an additional lesion of the prefrontal cortex (Klein et al., 2008). The precise description of the alterations that are taking place in the frontal cortex requires further studies. It might not only involve plastic changes in existing neurons, but also neurogenesis during the early age of the rat (Inta et al., 2008). A strong argument for this is that sodium butyrate has been shown to stimulate neurogenesis (Kim et al., 2009).

\section{MOTIVATION AND LEARNING}

Neonatal ventral hippocampal lesions rats weighed $10 \%$ less than their sham-operated littermates, as was the case in Chrapusta et al. (2003). The ventral hippocampus may be involved in regulation of food intake, especially when hedonic aspects are involved (Davidson et al., 2009). A contribution of the ventral hippocampus in the hedonic dimension of feeding would explain the lower body weight of NVHL rats. Nevertheless, participation of another behavioral abnormality or of some endocrine modification cannot be ruled out (Clifton et al., 1998) and are presently under investigation in our laboratory. Changes in the attitude of rats toward food may also explain the modification observed in the learning test used in this study. But aversively motivated learning was found to be impaired also in NVHL rats, indicating that it was probably a general aspect of learning that was modified by the lesion (Angst et al., 2007). Whatever the mechanism, our data highlight the fact that the modified behavior was completely countered by the phenylbutyrate treatment.

\section{PREFRONTAL HDAC ACTIVITY}

It is well known that indirect consequences of neonatal ventral hippocampal lesioning take place essentially in the prefrontal cortex (Goto and O'Donnell, 2004; Flores et al., 2005; Tseng et al., 2006, 2008; François et al., 2009). Adaptations to the prefrontal altered brain circuitry probably involve tremendous modifications in gene expression, in which epigenetic regulation is known to play a growing role (Colvis et al., 2005). When measuring total HDAC activity in the prefrontal cortex, we found it to be increased in lesioned vehicle-treated rats. It is striking that enhanced enzymatic activity was effective for as long as 4 months after the lesion was performed. Hence, it probably results from a novel equilibrium reached between acetylation and deacetylation processes in order to maintain the gene transcription at a crucial level for sustaining the functional impairment in the cortex. The treatment probably modifies the transcription of some specific genes. It remains to be determined though which genes are mostly affected. In a recent study, we demonstrated that genes encoding several HDACs are themselves under the control of HDAC inhibitors (Host et al., 2010). In turn, permanence of the enzymatic activity over a long period may account, at least partially, for the great stability of epigenetic regulations generally observed (Fraga et al., 2005). As expected, repeated treatment with a general HDAC inhibitor from the day following lesion until adulthood restored the activity in the prefrontal cortex back to the level found in sham-operated animals. In contrast, the inhibitor only slightly decreased HDAC activity in the prefrontal cortex of sham-operated rats, probably because the enzymes sensitive to phenylbutyrate are not sufficiently active in the cortex of normal adult rats for the inhibition to be discernible. It is worth noting that the increased HDAC activity found in NVHL rats parallels the increased activity found in post-mortem brain samples of schizophrenic patients (Sharma et al., 2008; Akbarian, 2010). This was correlated with a reduced expression in the prefrontal cortex of the GAD67 gene, which is known to be controlled by epigenetic mechanisms (Akbarian et al., 2005; Huang et al., 2007; Costa et al., 2009). If this type of regulation concerns a great many genes, it may help to explain the broad array of clinical, neuroanatomical, and molecular abnormalities reported in patients with schizophrenia, some of which are observable in NVHL rats.

\section{DATA INTERPRETATION LIMITS}

Some aspects of the work, which should not be over-interpreted, need further investigation. Firstly, like many other HDAC inhibitors, phenylbutyrate displays no selectivity toward the various class I and II HDACs; in addition, it may have other targets than the HDACs (Scaglia, 2010). Additional HDAC inhibitors displaying greater selectivity should be tested using a similar experimental strategy, or better, after having determined the critical duration of the treatment. A second remark concerns the period of life during which the treatment will be efficient. We hypothesized that similar results would be obtained when treating rats once they reached adulthood, as is the case in some other diseases (Fischer et al., 2007; Yildirim et al., 2008; Ricobaraza et al., 2009; Host et al., 2010; Kilgore et al., 2010; Peleg et al., 2010). We therefore conducted a similar experiment to the one presented here, except that the rats were only treated 1 week before and during the tests. While we reproduced exactly the effect of the lesion, phenylbutyrate-treated rats behaved like their vehicle-injected controls in all the tests (data not shown). Interestingly, that phenylbutyrate treatment was not efficient when given to adult animals argues against the possibility that the effects, observed when treatment followed immediately the lesioning, resulted from some trivial non-specific consequence of the inhibitor. Finally, another uncontrollable limiting factor arises from the interaction of the consequences of the treatment with determinant aspects of the experiments that cannot be avoided, such as maternal care (Wood et al., 2003) or stress (Laplante et al., 2004). 


\section{CONCLUSION}

We demonstrate that the mere inhibition of HDAC was sufficient to alleviate some, but not all the behavioral consequences due to lesioning the ventral hippocampus at an early age. Involvement of epigenetic regulations in the NVHL rat model is in line with the notion widely accepted among psychiatrists nowadays that schizophrenia results from genetic susceptibility combined with environmental influence, the latter operating through chromatin remodeling. Our results complement data showing that HDAC inhibitors may be useful to bring up brain plasticity to a point where it counters effects of developmental, traumatic, or degenerative damages to the brain (Fischer et al., 2007; Yildirim et al.,

\section{REFERENCES}

Akbarian, S. (2010). The molecular pathology of schizophrenia-focus on histone and DNA modifications. Brain Res. Bull. 83, 103-107.

Akbarian, S., Ruehl, M. G., Bliven, E., Luiz, L. A., Peranelli, A. C., Baker, S. P., Roberts, R. C., Bunney, W. E. Jr., Conley, R. C., Jones, E. G., Tamminga, C. A., and Guo, Y. (2005). Chromatin alterations associated with downregulated metabolic gene expression in the prefrontal cortex of subjects with schizophrenia. Arch. Gen. Psychiatry 62, 829-840.

Angst, M. J., Macedo, C. E., Guiberteau, T., and Sandner, G. (2007). Alteration of conditioned emotional response and conditioned taste aversion after neonatal ventral hippocampus lesions in rats. Brain Res. 1143, 183-192.

Beninger, R. J., Tuerke, K. J., Forsyth, J. K., Giles, A., Xue, L., Boegman, R. J., and Jhamandas, K. (2009). Neonatal ventral hippocampal lesions in male and female rats: effects on water maze, locomotor activity, plus-maze and prefrontal cortical GABA and glutamate release in adulthood. Behav. Brain Res. 202, 198-209.

Bertrand, J. B., Langlois, J. B., Bégou, M., Volle, J., Brun, P., d'Amato, T., Saoud, M., and Suaud-Chagny, M. F. (2010). Longitudinal MRI monitoring of brain damage in the neonatal ventral hippocampal lesion rat model of schizophrenia. Hippocampus 20, 264-278.

Broersen, L. M. (2000). Attentional processes and learning and memory in rats: the prefrontal cortex and hippocampus compared. Prog. Brain Res. 126, 79-94.

Cahn, W., Rais, M., Stigter, F. P., van Haren, N. E., Caspers, E., Hulshoff Pol, H. E., Xu, Z., Schnack, H. G., and Kahn, R. S. (2009). Psychosis and brain volume changes during the first five years of schizophrenia. Eur. Neuropsychopharmacol. 19, 147-151.

Chrapusta, S. J., Egan, M. F., Wyatt, R. J., Weinberger, D. R., and Lipska,
B. K. (2003). Neonatal ventral hippocampal damage modifies serum corticosterone and dopamine release responses to acute footshock in adult Sprague-Dawley rats. Synapse 15, 270-277.

Clifton, P.G., Vickers, S. P., and Somerville, E.M. (1998). Little and often: ingestive behavior patterns following hippocampal lesions in rats. Behav. Neurosci. 112, 502-511.

Colvis, C. M., Pollock, J. D., Goodman, R. H., Impey, S., Dunn, J., Mandel, G., Champagne, F. A., Mayford, M., Korzus, E., Kumar, A., Renthal, W., Theobald, D. E., and Nestler, E. J. (2005). Epigenetic mechanisms and gene networks in the nervous system. J. Neurosci. 25, 10379-10389.

Costa, E., Chen, Y., Dong, E., Grayson, D. R., Kundakovic, M., Maloku, E., Ruzicka, W., Satta, R., Veldic, M., Zhubi, A., and Guidotti, A. (2009). GABAergic promoter hypermethylation as a model to study the neurochemistry of schizophrenia vulnerability. Expert Rev. Neurother. 9, 87-98.

Dash, P. K., Orsi, S. A., and Moore, A. N. (2009). Histone deacetylase inhibition combined with behavioral therapy enhances learning and memory following traumatic brain injury. Neuroscience 163, 1-8.

Davidson, T. L., Chan, K., Jarrard, L. E., Kanoski, S. E., Clegg, D. J., and Benoit, S. C. (2009). Contributions of the hippocampus and medial prefrontal cortex to energy and body weight regulation. Hippocampus 19, 235-252.

Degroot, A., and Treit, D. (2004). Anxiety is functionally segregated within the septo-hippocampal system. Brain Res. 1001, 60-71.

Ellenbroek, B. A., and Cools, A. R. (2002). Apomorphine susceptibility and animal models for psychopathology: genes and environment. Behav. Genet. 32, 349-361.

Featherstone, R. E., Kapur, S., and Fletcher, P.J. (2007). The amphetamine-induced sensitized state as a model of schizophrenia. Prog. Neuropsychopharmacol. Biol. Psychiatry 31, 1556-1571.

2008; Ricobaraza et al., 2009; Kilgore et al., 2010; Peleg et al., 2010). Considering the neurodevelopmental hypothesis of schizophrenia, the data support the concept that chromatin remodeling may be helpful for preventing some aspects of the disease.

\section{ACKNOWLEDGMENTS}

We would like to thank Julien Gobaille and Jacques Knobloch for having taken well care of the dams and pups until they reached adulthood. The RMI images were obtained by courtesy of the "Plateforme d'Imagerie in vivo de Strasbourg, LINC, campus médecine," IFR 37 de Neurosciences de Strasbourg. Help with the English language was provided by Mrs. Gillian Wakenhut.

Fischer, A., Sananbenesi, F., Wang, X., Dobbin, M., and Tsai, L. H. (2007). Recovery of learning and memory is associated with chromatin remodelling. Nature 447, 178-182.

Flores, G., Alquicer, G., Silva-Gómez, A B., Zaldivar, G., Stewart, J., Quirion, R. and Srivastava, L. K. (2005). Alterations in dendritic morphology of prefrontal cortical and nucleus accumbens neurons in post-pubertal rats after neonatal excitotoxic lesions of the ventral hippocampus. Neuroscience 133, 463-470.

Flores, G., Barbeau, D., Quirion, R., and Srivastava, L. K. (1996). Decreased binding of dopamine D3 receptors in limbic subregions after neonata bilateral lesion of rat hippocampus. $J$. Neurosci. 16, 2020-2026.

Fraga, M. F., Ballestar, E., Paz, M. F., Ropero, S., Setien, F., Ballestar, M. L., Heine-Suñer, D., Cigudosa, J. C., Urioste, M., Benitez, J., BoixChornet, M., Sanchez-Aguilera, A. Ling, C., Carlsson, E., Poulsen, P. Vaag, A., Stephan, Z., Spector, T. D., Wu, Y. Z., Plass, C., and Esteller, M. (2005). Epigenetic differences arise during the lifetime of monozygotic twins. Proc. Natl. Acad. Sci. U.S.A. 102 10604-10609.

François, J., Ferrandon, A., Koning, E. Angst, M. J., Sandner, G., and Nehlig, A. (2009). Selective reorganization of GABAergic transmission in rats with neonatal ventral hippocampus lesion. Int. J. Neuropsychopharmacol. 9, 1-14.

Gavin, D. P., and Sharma, R. P. (2010). Histone modifications, DNA methylation, and Schizophrenia. Neurosci. Biobehav. Rev. 34, 882-888.

Goto, Y., and O'Donnell, P. (2004). Prefrontal lesion reverses abnormal mesoaccumbens response in an animal model of schizophrenia. Biol. Psychiatry 55, 172-176.

Halim, N. D., and Swerdlow, N. R. (2000) Distributed neurodegenerative changes 2-28 days after ventral hippocampal excitotoxic lesions in rats. Brain Res. 873, 60-74.
Holland, P.C., and Petrovich, G. D. (2005). A neural systems analysis of the potentiation of feeding by conditioned stimuli. Physiol. Behav. 86, 747-761.

Host, L., Dietrich, J. B., Carouge, D., Aunis, D., and Zwiller, J. (2010). Cocaine selfadministration alters the expression of chromatin-remodelling proteins; modulation by histone deacetylase inhibition. J. Psychopharmacol. doi: 10.1177/0269881109348173. [Epub print of ahead].

Huang, H. S., Matevossian, A., Whittle, C. Kim, S. Y., Schumacher, A., Baker, S. P., and Akbarian, S. (2007). Prefrontal dysfunction in schizophrenia involves mixed-lineage leukemia 1-regulated histone methylation at GABAergic gene promoters. J. Neurosci. 27, 11254-11262.

Inta, D., Alfonso, J., von Engelhardt, J., Kreuzberg, M. M., Meyer, A. H., van Hooft, J. A., and Monyer, H. (2008). Neurogenesis and widespread forebrain migration of distinct GABAergic neurons from the postnatal subventricular zone. Proc. Natl. Acad. Sci. U.S.A. 105, 20994-20999.

Kilgore, M., Miller, C. A., Fass, D. M., Hennig, K. M., Haggarty, S. J., Sweatt, J. D., and Rumbaugh, G. (2010). Inhibitors of class 1 histone deacetylases reverse contextual memory deficits in a mouse model of Alzheimer's disease. Neuropsychopharmacology 35 , 870-880.

Kim, H. J., Leeds, P., and Chuang, D. M. (2009). The HDAC inhibitor, sodium butyrate, stimulates neurogenesis in the ischemic brain. J. Neurochem. 110, 1226-1240.

Klein, S., Koch, M., and Schwabe, K. (2008). Neuroanatomical changes in the adult rat brain after neonatal lesion of the medial prefrontal cortex. Exp. Neurol. 209, 199-212.

Laplante, F., Stevenson, C. W., Gratton, A., Srivastava, L. K., and Quirion, R. (2004). Effects of neonatal ventral hippocampal lesion in rats on stressinduced acetylcholine release in the prefrontal cortex. J. Neurochem. 91, 1473-1482. 
Lepekhina, L. M., and Tsitsurina, E. A. (2007). Stereotyped behavior in the ontogeny of rats. Bull. Exp. Biol. Med. 144, 349-351.

Lipska, B. K. (2004). Using animal models to test a neurodevelopmental hypothesis of schizophrenia. J. Psychiatry Neurosci. 29, 282-286.

Lipska, B. K., Al-Amin, H. A., and Weinberger, D. R. (1998). Excitotoxic lesions of the rat medial prefrontal cortex. Effects on abnormal behaviors associated with neonatal hippocampal damage. Neuropsychopharmacology 19, 451-464.

Lopez-Rodas, G., Brosch, G., Georgieva, E. I., Sendra, R., Franco, L., and Loidl, P. (1993). Histone deacetylase. A key enzyme for the binding of regulatory proteins to chromatin. FEBS Lett. 317 , 175-180.

Macedo, C. E., Angst, M. J., Guiberteau, T., Brasse, D., O'Brien, T., and Sandner, G. (2010).Acoustic hypersensitivity in adult rats after neonatal ventral hippocampus lesions. Behav. Brain Res. 207, 161-168.

Macedo, C. E., Sandner, G., Angst, M. J., and Guiberteau, T. (2008). Rewarded associative and instrumental conditioning after neonatal ventral hippocampus lesions in rats. Brain Res. 215, 190-199.

Nauta, W. J. (1971). The problem of the frontal lobe: a reinterpretation. $J$. Psychiatr. Res. 8, 167-187.

Peleg, S., Sananbenesi, F., Zovoilis, A., Burkhardt, S., Bahari-Javan, S., AgisBalboa, R. C., Cota, P., Wittnam, J. L., Gogol-Doering, A., Opitz, L., SalinasRiester, G., Dettenhofer, M., Kang, H., Farinelli, L., Chen, W., and Fischer, A. (2010). Altered histone acetylation is associated with age-dependent memory impairment in mice. Science 328, 753-756.

Pellow, S., and File, S.E. (1986). Anxiolytic and anxiogenic drug effects on exploratory activity in an elevated plus-maze: a novel test of anxiety in the rat. Pharmacol. Biochem. Behav. 24, 525-529.

Ricobaraza, A., Cuadrado-Tejedor, M., Pérez-Mediavilla, A., Frechilla, D., Del Río, J., and García-Osta, A. (2009). Phenylbutyrate ameliorates cognitive deficit and reduces tau pathology in an Alzheimer's disease mouse model. Neuropsychopharmacology 34 , 1721-1732.

Rolls, E. T. (2000). Memory systems in the brain. Annu. Rev. Psychol. 51, 599-630.

Romieu, P., Host, L., Gobaille, S., Sandner, G., Aunis, D., and Zwiller, J. (2008). Histone deacetylase inhibitors decrease cocaine but not sucrose selfadministration in rats. J. Neurosci. 28 , 9342-9348.

Roth, T. L., Lubin, F. D., Sodhi, M., and Kleinman, J. E. (2009). Epigenetic mechanisms in schizophrenia. Biochim. Biophys. Acta 1790, 869-877.

Sandner, G., Angst, M. J., Guiberteau, T., Guignard, B., and Brasse, D. (2010). MRI and X-ray scanning images of the brain of 3, 6 and 9 months old rats with bilateral neonatal ventral hippocampus lesions. Neuroimage $53,44-50$.

Scaglia, F. (2010). New insights in nutritional management and amino acid supplementation in urea cycle disorders. Mol. Genet.Metab. 100(Suppl. 1), S72-S76.

Schmitt, K. C., and Reith, M. E. (2010). Regulation of the dopamine transporter: aspects relevant to psychostimulant drugs of abuse. Ann. N. Y. Acad. Sci. 1187, 316-340.

Schroeder, H., Grecksch, G., Becker, A., Bogerts, B., and Hoellt, V. (1999). Alterations of the dopaminergic and glutamatergic neurotransmission in adult rats with postnatal ibotenic acid hippocampal lesion.
Psychopharmacology (Berl.) 145, 61-66.

Shah, A. A., and Treit, D. (2003). Excitotoxic lesions of the medial prefrontal cortex attenuate fear responses in the elevated-plus maze, social interaction and shock probe burying tests. Brain Res. 969, 183-194.

Sharma, R. P., Grayson, D. R., and Gavin, D. P. (2008). Histone deacetylase 1 expression is increased in the prefrontal cortex of schizophrenia subjects: analysis of the National Brain Databank microarray collection. Schizophr. Res. 98, 111-117.

Szyf, M., McGowan, P., and Meaney, M. J. (2008). The social environment and the epigenome. Environ. Mol. Mutagen. 49, 46-60.

Tseng, K. Y., Amin, F., Lewis, B. L., and O’Donnell, P. (2006). Altered prefrontal cortical metabolic response to mesocortical activation in adult animals with a neonatal ventral hippocampal lesion. Biol. Psychiatry 60 585-590.

Tseng, K. Y., Chambers, R. A., and Lipska, B. K. (2009). Neonatal ventral hippocampal lesion as a heuristic neurodevelopmental model of schizophrenia. Behav. Brain Res. 204, 295-305.

Tseng, K. Y., Lewis, B. L., Hashimoto, T., Sesack, S. R., Kloc, M., Lewis, D. A., and O'Donnell, P. (2008). A neonatal ventral hippocampal lesion causes functional deficits in adult prefrontal cortical interneurons. J. Neurosci. 28 12691-12699.

Wood, G. K., Lipska, B. K., and Weinberger, D. R. (1997). Behavioral changes in rats with early ventral hippocampal damage vary with age at damage. Brain Res. Dev. Brain Res. 101, 17-25.

Wood, G. K., Quirion, R., and Srivastava, L. K. (2003). Early environment contributes to developmental disruption of MPFC after neonatal ventral hippocampal lesions in rats. Synapse 50, 223-232.

Yildirim, F., Gertz, K., Kronenberg, G., Harms, C., Fink, K. B., Meisel, A., and Endres, M. (2008). Inhibition of histone deacetylation protects wildtype but not gelsolin-deficient mice from ischemic brain injury. Exp. Neurol. 210, 531-542.

Conflict of Interest Statement: This work was funded by INSERM and by the University of Strasbourg, France. These institutional funding administrations played no further role in the design of the study, in the collection, analysis, and interpretation of data, writing of the report, or in the decision to submit the manuscript for publication. The authors declare that, except for income received from our primary employer, no financial support or compensation has been received from any individual or corporate entity over the past 3 years for research or professional service and that there are no personal financial holdings that could be perceived as constituting a potential conflict of interest.

Received: 27 September 2010; accepted: 17 December 2010; published online: 07 January 2011.

Citation: Sandner G, Host L, Angst M-J, Guiberteau T, Guignard B and Zwiller J (2011) The HDACinhibitor phenylbutyrate reverses effects of neonatal ventral hippocampal lesion in rats. Front. Psychiatry 1:153. doi: 10.3389/fpsyt.2010.00153

This article was submitted to Frontiers in Schizophrenia, a specialty of Frontiers in Psychiatry.

Copyright $\odot 2011$ Sandner, Host, Angst, Guiberteau, Guignard and Zwiller. This is an open-access article subject to an exclusive license agreement between the authors and the Frontiers Research Foundation, which permits unrestricted use, distribution, and reproduction in any medium, provided the original authors and source are credited. 